\title{
PREVALÊNCIA DE PERDA AUDITIVA E FATORES ASSOCIADOS NA POPULAÇÃO IDOSA DE LONDRINA, PARANÁ: ESTUDO PRELIMINAR
}

\section{Prevalence of hearing loss and associated factors in elderly population in Londrina, Paraná: preliminary study}

\author{
Caroline Meneses ${ }^{(1)}$, Mariana Peretti Mário ${ }^{(2)}$, Luciana Lozza de Moraes Marchori (3), \\ Juliana Jandre Melo ${ }^{(4)}$, Eliane Regina Ferreira Sernache de Freitas ${ }^{(5)}$
}

\begin{abstract}
RESUMO
Objetivo: estimar a prevalência de Perda Auditiva (PA), identificando os fatores associados em pacientes idosos de Londrina - PR. Métodos: estudo transversal realizado em amostra populacional de 61 indivíduos com idade igual ou superior a 60 anos, em 2009, através de anamnese e avaliação audiológica. Aplicou-se o teste do Qui-quadrado e o do Risco Relativo com 95\% de confiança e valor de $p<0,05$ para as análises univariadas entre PA e fatores associados. Resultados: de toda a amostra $87,70 \%$ apresentaram algum tipo de PA ou rebaixamento de frequências. Destes indivíduos, o tipo de PA de maior prevalência foi a PA sensorioneural, com $63,93 \%$ de ocorrência. Entre os hipertensos, $88,88 \%$ tiveram PA, sendo este um aumento de 8 chances a mais de hipertensos apresentarem PA. Dos diabéticos, $90 \%$ tiveram PA, ou seja, um aumento de 9 chances a mais de diabéticos apresentarem PA. Dos consumidores de álcool, 92,30\% apresentaram PA, sendo assim, os consumidores de álcool apresentaram 12 chances a mais de ter PA. A partir da amostra de fumantes, ex-fumantes ou fumantes passivos, $87,09 \%$ apresentaram PA, tendo assim, 9 chances a mais de terem PA quando relacionados com não fumantes. Conclusão: a PA sensorioneural é a mais encontrada, sendo progressiva e proporcionalmente aumentada com o envelhecimento nesta população. Os fatores de risco hipertensão, diabetes, consumo alcoólico e fumo podem predispor as perdas auditivas, principalmente quando associados com a presbiacusia.
\end{abstract}

DESCRITORES: Perda Auditiva; Fatores de Risco; Estudos Transversais.

(1) Fonoaudióloga; Voluntária de iniciação científica do Curso de Fonoaudiologia da Universidade Norte do Paraná, UNOPAR, Londrina, PR, Brasil.

(2) Fonoaudióloga; Voluntária de iniciação científica do Curso de Fonoaudiologia da Universidade Norte do Paraná, UNOPAR, Londrina, PR, Brasil.

(3) Fonoaudióloga; Docente da Universidade Norte do Paraná, UNOPAR, Londrina, PR, Brasil; Doutora em Medicina e Ciências da Saúde pela Universidade Estadual de Londrina.

(4) Fonoaudióloga; Docente da Universidade Norte do Paraná, UNOPAR, Londrina, PR, Brasil; Mestre em Distúrbios da Linguagem pela Pontifícia Universidade Católica de São Paulo.

(5) Fisioterapeuta; Docente da Universidade Norte do Paraná, UNOPAR, Londrina, PR, Brasil; Doutora em Ciências da Saúde pela Universidade Federal de São Paulo/Escola Paulista de Medicina.

Conflito de interesses: inexistente

\section{INTRODUÇÃO}

Atualmente, há uma crescente elevação da expectativa média de vida, ligada a uma diminuição gradual das taxas de mortalidade e natalidade ${ }^{1,2}$.

O envelhecimento populacional é um fenômeno global, sendo que provavelmente o número de pessoas com mais de 60 anos irá crescer mais de $300 \%$ nos próximos 50 anos, passando de 606 milhões em 2000 para aproximadamente dois bilhões em 2050, dos quais 1,6 bilhões virão dos países menos desenvolvidos e em desenvolvimento; só o Brasil contribuirá com 58 milhões ${ }^{3}$.

No Brasil, a população idosa está aumentando e já responde por $10,2 \%$ da população total. Londrina, hoje, tem 46.325 habitantes com mais de 60 anos 
de idade ${ }^{2,4-9}$. Possivelmente este quadro se deva ao avanço tecnológico científico e das melhores condições médicas, higiênico-sanitárias e de saneamento básico, mesmo que ainda longe das ideais ${ }^{10}$.

Nas condições atuais da sociedade, os idosos são desvalorizados, considerados como incapazes por sua baixa produtividade devido às limitações naturais e ao declínio de várias funções vitais como das acuidades auditiva e visual, da diminuição das sensibilidades tátil e dolorosa determinadas por sua idade ${ }^{10}$.

Esta situação vem despertando o interesse de vários setores da sociedade como profissionais da área da saúde que têm se empenhado em se interar dos diversos distúrbios que acometem esta faixa etária para melhor atendê-los, proporcionando maior conforto e adaptação às modificações que ocorrem no processo de envelhecimento ${ }^{5}$.

A fonoaudiologia é uma destas áreas que estão contribuindo para a prevenção e tratamento das alterações provenientes do envelhecimento, buscando atender a população idosa e melhorando sua qualidade de vida ${ }^{2,11}$.

Apesar de o fato da expectativa de vida ter aumentado isto não significa que as condições de vida para o idoso também tenham melhorado ${ }^{10}$. Das alterações sensoriais sofridas por estes indivíduos, a diminuição da acuidade auditiva representa uma das principais causas de isolamento social para o idoso, sendo que a perda auditiva configurase como a alteração que produz maior impacto na comunicação e na sua vida psicossocial podendo levar também a depressão e a privação das atividades de vida diária ${ }^{5,12}$.

A perda auditiva é a terceira condição crônica mais prevalente entre os idosos, ficando atrás apenas da artrite e da hipertensão arterial, com uma ligeira tendência para os homens apresentarem maior prevalência do que as mulheres ${ }^{2,13}$.

Segundo dados da ASHA (American Speech Language - Hearing Association), atualmente, 28 milhões de indivíduos nos Estados Unidos apresentam alguma perda auditiva, sendo que $80 \%$ dos mesmos, irreversíveis. Mostram também que $4,6 \%$ dos indivíduos entre 18 e 44 anos têm perda auditiva, enquanto que $14 \%$ dos indivíduos de meia idade, entre 45 e 64 anos e $54 \%$ da população acima dos 65 anos apresentam alguma perda. Isto se deve a inúmeros fatores, tais como ruído intenso e/ou contínuo, inalação de substâncias tóxicas, ingestão de medicamentos ototóxicos, alterações metabólicas e circulatórias, além de infecções, traumas de várias naturezas e hereditariedade ${ }^{14}$.

Alguns estudos justificam que a surdez sensorioneural que acontece com a idade tem relação com uma insuficiência microcirculatória decorrente de uma oclusão vascular por embolia, hemorragia ou vasoespasmo e que estes, por sua vez, seriam decorrentes de uma síndrome de hiperviscosidade ou microangiopatia por diabetes ou hipertensão, sendo que a hipertensão poderia através desses fatores histopatológicos, provocarem perda de audição sensorioneural ${ }^{15}$.

Com o avanço da idade cresce o número de doenças crônicas sendo a hipertensão arterial sistêmica e a perda auditiva de grande prevalência na população idosa ${ }^{16}$. Muitas pesquisas foram realizadas acerca da associação entre hipertensão arterial e perda auditiva. Algumas destas pesquisas verificaram a existência da associação significativa entre elas ${ }^{15,17}$ e sugerem que a hipertensão arterial age como fator de aceleração da degeneração do aparelho auditivo proveniente da idade ${ }^{15}$. Outros estudos não comprovaram maior déficit auditivo em hipertensos ${ }^{16}$.

Em relação a pacientes com diabetes mellitus, estes frequentemente apresentam sintomas como tontura, zumbidos e hipoacusia ${ }^{18}$.

Diabetes mellitus, dentro dos distúrbios do metabolismo da glicose, é a afecção mais comumente relacionada a distúrbios auditivos ${ }^{18}$.

A presença de sintomas auditivos associados a diabetes mellitus é discutida a seis décadas ${ }^{19}$, entretanto esta associação entre perda auditiva e diabetes mellitus ainda é bastante controversa ${ }^{18,19}$. Geralmente a perda auditiva é do tipo sensorioneural, confundindo-se com presbiacusia, principalmente por ocorrer em pacientes acima dos 40 anos de idade ${ }^{18}$.

Outro fator de risco citado pela literatura como associado à perda auditiva é o consumo alcoólico.

Diversas drogas ototóxicas causam efeitos nocivos ao ser humano, como zumbido, várias formas de deficiências auditivas e vertigem ${ }^{20}$. $\mathrm{O}$ alcoolismo é considerado a toxomania de maior relevância na escala mundial ${ }^{20,21}$ e a perda auditiva sensorioneural relacionada ao uso abusivo do álcool vem sendo estudada, mas seus resultados ainda são contraditórios ${ }^{21}$, pois apesar de serem encontrados na literatura estudos que constatam o efeito nocivo do álcool na audição ${ }^{20,21}$ muitas vezes este fator está associado à idade e por isto sua comprovação ainda é discutida.

O tabagismo também tem sido considerado como um fator de risco otológico. Atualmente o cigarro é considerado a principal causa de enfermidades evitáveis e incapacidades prematuras e chegará a ser a primeira causa de morte evitável no final do século XXI ${ }^{22}$.

O cigarro pode afetar a audição pelo efeito do mecanismo antioxidante, ou pela supressão 
vascular do sistema auditivo ${ }^{22}$. De acordo com a pesquisa de análise dos potenciais evocados auditivos em fumantes, o tabagismo parece afetar as vias auditivas centrais nas regiões de tronco encefálico baixo e subcorticais, não afetando a cognição ${ }^{22}$.

Diante de tantos desafios naturais e sociais enfrentados por este grupo populacional, ainda precisam enfrentar a dificuldade de comunicar-se o que impede o pleno desempenho de seu papel na sociedade comprometendo seu relacionamento com seus familiares e amigos ${ }^{10,12}$.

Esta perda da sensibilidade auditiva resultante do envelhecimento é conhecida como presbiacusia $^{23}$. Refere-se ao resultado de um longo período de danos ao sistema auditivo, de uma variedade de degeneração fisiológica incluindo prejuízos causados pela exposição ao ruído, agentes ototóxicos e outras desordens causadas por demais tratamentos médicos $1,11,23,24$.

A presbiacusia é uma doença crônica de etiologia multifatorial ${ }^{6}$, iniciando-se a partir dos 20/30 anos de idade, podendo se tornar um incômodo a partir dos 40/50 anos ${ }^{6}$.

Idosos presbiacúsicos experimentam uma redução na sensibilidade auditiva e uma redução na inteligibilidade da fala, bem como o rebaixamento do limiar de altas frequências e a habilidade de recordar sentenças longas, comprometendo seriamente o processo de comunicação verbal ${ }^{2,12,23,25,26}$.

Durante o exame médico tradicional nestes indivíduos, a audição não está incluída nas avaliações. Uma triagem auditiva que inclui o exame audiométrico seria de grande valia para a identificação precoce de perda ou declínio auditivo, já que os pacientes, de modo geral, não têm consciência da instalação desta alteração e por isto a aplicação de questionários investigativos não são totalmente confiáveis ${ }^{13}$.

A partir destas informações, o objetivo desta pesquisa foi estimar a prevalência de perda auditiva, identificando os fatores associados em pacientes idosos do município de Londrina.

\section{MÉTODOS}

Foi realizado um estudo transversal com inclusão de indivíduos com idade superior a 60 anos, de ambos os sexos, com vida independente, que estavam classificados nos níveis 3 e 4 do Status Funcional proposto por Spirduso (2005) ${ }^{27}$, e que aceitaram participar voluntariamente do estudo.

De uma população de 43.610 idosos cadastrados nas 38 UBSs da zona urbana de Londrina, a amostra calculada foi de 343 indivíduos. Levandose em consideração perdas amostrais em torno de
$20 \%$, em alguns estudos realizados com idosos, a amostra foi elevada para 414 sujeitos. A amostragem foi definida de forma aleatória estratificada, levando-se em consideração o gênero e as cinco regiões do município; sendo: $15 \%$ (62) da região central, $27 \%$ (111) da região norte, 23\% (94) da região sul, $19 \%$ (78) da região leste e $16 \%$ (69) da região oeste. Para o cálculo foi considerado um erro amostral de 5\%. Deste número, este estudo preliminar visou verificar o resultado dos 61 primeiros idosos avaliados.

A pesquisa diagnóstica para avaliação da audição consistiu de anamnese audiológica utilizada na rotina de atendimentos no setor de audiologia da UNOPAR, baseada no protocolo para anamnese de Katz ${ }^{28}$ e da audiometria tonal limiar considerada o padrão ouro para avaliar limiar auditivo em adultos ${ }^{29}$ anotado na ficha para audiometria tonal utilizada na rotina de atendimentos no setor de audiologia da UNOPAR e passada no banco de dados do programa Winaudio para ser armazenada e impressa para o paciente.

Como sistemática operacional para a execução do projeto foi realizada anamnese audiológica seguida de audiometria tonal limiar.

A variável dependente foi à presença de perda auditiva. As variáveis independentes foram: idade, fumo e álcool, as alterações sistêmicas hipertensão arterial e diabetes mellitus. As variáveis foram apresentadas de forma descritiva em números absolutos e proporções, estimando-se a prevalência.

Foi realizado estudo com os testes Qui-quadrado e Risco Relativo para verificar as possíveis diferenças entre os fatores de risco e os indivíduos que apresentaram e os que não apresentaram perda auditiva.

Para a análise univariada, os dados quantitativos foram categorizados em idade, hipertensão arterial, diabetes mellitus, álcool e fumo e foi aplicado o teste do Qui-quadrado nas categorias: idade entre 70-79 anos, 80-89 anos e acima de 90 anos; e o teste do Risco Relativo nas categorias: idade entre 60-69 anos; diabetes mellitus; hipertensão arterial; fumo e álcool. Também foram quantificados os resultados dos limiares auditivos encontrados em toda amostra para identificar o tipo de perda de maior ocorrência.

Foram considerados para as análises univariadas $p<0,01$ e para a inclusão no modelo final para o teste Qui-quadrado e para o Risco Relativo o valor de $p<0,05$, ambos com intervalo com $95 \%$ de confiança.

Como critério para perda auditiva foi usado a classificação de Davis e Silverman ${ }^{25}$. A idade foi categorizada em quatro extratos: de 60 a 69 anos, 
de 70 a 79 anos, de 80 a 89 anos e acima de 90 anos.

O consumo de álcool foi considerado como: excessivo (consumo em finais de semana com embriaguez frequente e/ou consumo diário com ou sem embriaguez, consumo entre 2 a 4 vezes por semana e semanalmente) ou moderado (frequência de bebida de 3 vezes ao mês ou mensais) e os demais foram classificados como não consumidores.

O consumo de cigarros foi classificado como: fumantes, ex-fumantes, fumantes passivos (com contato diário) e não fumantes; sendo que para o teste de Risco relativo foram divididos em 2 grupos: grupo A (fumantes, ex-fumantes e fumantes passivos) e grupo $B$ (não fumantes).

Para a classificação entre perda auditiva e hipertensão arterial foi utilizada a amostra de indivíduos que apresentaram hipertensão arterial e quantificados os com perda auditiva e os sem perda auditiva. Foram respeitadas as normas estabelecidas pelo III Consenso Brasileiro de Hipertensão Arterial de $1998{ }^{30}$. O mesmo procedimento foi utilizado para o fator de risco diabetes mellitus, onde foi aplicado o "Questionário de Comorbidades" utilizado no Estudo sobre o envelhecimento em Londrina EELO.

A análise e aprovação do projeto, PP/ 0063/09, bem como do termo de consentimento pela resolução 196/96-CNS.

\section{RESULTADOS}

A amostra foi constituída por 61 indivíduos em idade entre 60 e 95 anos, sendo 24 homens e 37 mulheres. Destes, $59,01 \%$ da amostra apresentaram hipertensão arterial; 32,78\% apresentaram diabetes mellitus; $21,31 \%$ foram classificados como consumidores excessivos de álcool e 51,7\% foram classificados como fumantes ex-fumantes ou fumantes passivos com contato diário com fumantes.

De toda a amostra (61 indivíduos - 122 orelhas), $87,70 \%$ (107 orelhas) apresentaram algum tipo de perda auditiva ou rebaixamento de frequências e $12,30 \%$ (15 orelhas) apresentaram os limiares auditivos dentro dos padrões de normalidade, sendo que estes $12,30 \%$ pertenciam à categoria entre 60-69 anos. Não foram encontrados limiares normais a partir dos 70 anos de idade. Através do teste estatístico do Risco relativo, verificou-se que existe aumento absoluto do risco de $75,40 \%$ de indivíduos com mais de 60 anos sofrerem algum tipo de perda auditiva, sendo que esta estimativa indica que existem em média 7,13 chances a mais de ter perda auditiva após os 60 anos. Este resultado foi altamente significativo, apresentando $p<$ 0,0001 (Tabela 1).

A partir desta mesma amostra, como elucidado na Tabela 2, foram considerados os tipos de perda encontrados entre indivíduos com mais de 60 anos de idade, a fim de identificar o tipo de perda de maior prevalência nesta faixa etária. De toda a amostra $12,30 \%$ (15 orelhas/ 60-69 anos) obtiveram limiares normais, $22,13 \%$ (27 orelhas) limiares normais com frequências rebaixadas $(6000$ e $8000 \mathrm{~Hz}), 63,93 \%$ (78 orelhas) perda auditiva sensorioneural e 1,64\% (2 orelhas) outros tipos de perda.

A amostra total foi classificada em diferentes faixas etárias: 60 a 69 anos, 70 a 79 anos, 80 a 89 anos e acima de 90 anos, como pode ser observado na Tabela 3.

Tabela 1 - Pesquisa de perda auditiva em amostra total

\begin{tabular}{lcc}
\hline \multicolumn{3}{c}{ Limiares Auditivos } \\
\hline Com perda e sem perda auditiva & Orelhas & Porcentagem \\
\hline Com perda auditiva ou rebaixamento de frequências (107 orelhas) & 107 & $87,70 \%$ \\
Sem perda auditiva (15 orelhas) & 15 & $12,30 \%$ \\
\hline
\end{tabular}

Tabela 2 - Perda auditiva de maior prevalência em amostra total

\begin{tabular}{lccc}
\hline \multicolumn{3}{c}{ Limiares Auditivos $-\mathbf{p}<\mathbf{0 , 0 0 0 1}$} & \\
\hline Classificação Auditiva & Orelhas & Porcentagem \\
\hline Limiares normais (15 orelhas) & 15 & $12,30 \%$ \\
Limiares normais com frequência rebaixada (27 orelhas) & 27 & $22,13 \%$ \\
Perda auditiva sensorioneural (78 orelhas) & 78 & $63,93 \%$ \\
Outros tipos de perda auditiva (2 orelhas) & 2 & $1,64 \%$ \\
\hline
\end{tabular}

Rev. CEFAC, São Paulo 
Tabela 3 - Limiares auditivos em diferentes faixas etárias

\begin{tabular}{lcccc}
\hline \multicolumn{4}{c}{ Limiares Auditivos e Idade } \\
\hline Parecer audiológico & $\begin{array}{c}\mathbf{6 0 - 6 9} \text { anos } \\
\mathbf{p}<\mathbf{0 , 0 0 0 1}\end{array}$ & $\begin{array}{c}\mathbf{7 0 - 7 9} \text { anos } \\
\mathbf{p}<\mathbf{0 , 0 0 0 1}\end{array}$ & $\begin{array}{c}\mathbf{8 0 - 8 9} \text { anos } \\
\mathbf{p}=\mathbf{0 , 7 0 4 7}\end{array}$ & $\begin{array}{c}\text { Acima } \\
\text { de } \mathbf{9 0} \text { anos } \\
\mathbf{p = 0 , 5 4 6 5}\end{array}$ \\
\hline Limiares normais & $22 \%$ & & & \\
Limiares normais exceto 6000 e $8000 \mathrm{~Hz}$ & $28 \%$ & $18 \%$ & & $50 \%$ \\
Perda sensorioneural & $28 \%$ & $43 \%$ & $17 \%$ & \\
Perda sensorioneural de grau leve & $13 \%$ & $18 \%$ & & $50 \%$ \\
Perda mista de grau leve & $7 \%$ & $3 \%$ & $33 \%$ & \\
Perda sensorioneural de grau moderado & & $7 \%$ & & \\
Perda sensorioneural de grau severo & $2 \%$ & $14 \%$ & & \\
Perda mista de grau severo & & $2 \%$ & & \\
Perda sensorioneural de grau profundo & & &
\end{tabular}

$\mathrm{Na}$ audiometria tonal limiar foi encontrado os seguintes achados audiométricos:

Nos indivíduos entre 60 a 69 anos (34 pessoas/ 68 orelhas): $22 \%$ limiares auditivos dentro dos padrões de normalidade; $28 \%$ limiares auditivos dentro dos padrões de normalidade, com queda em 6000 e $8000 \mathrm{~Hz} ; 28 \%$ perda auditiva sensorioneural; $13 \%$ perda auditiva sensorioneural de grau leve; $7 \%$ perda auditiva sensorioneural de grau moderado e $2 \%$ perda auditiva mista de grau severo. Nesta faixa etária, alguns indivíduos apresentaram limiares auditivos normais, por isto foi possível realizar o teste de Risco Relativo, cruzando os dados de perda auditiva e de limiares normais. Através desta técnica conclui-se que nesta faixa de idade, existe um aumento absoluto do risco de $55,88 \%$ de indivíduos entre 60 e 69 anos sofrerem algum tipo de perda auditiva.

$\mathrm{Na}$ faixa etária entre 70 a 79 anos (22 pessoas/ 44 orelhas): $18 \%$ limiares auditivos dentro dos padrões de normalidade, com queda em 6000 e $8000 \mathrm{~Hz} ; 43 \%$ perda auditiva sensorioneural; $18 \%$ perda auditiva sensorioneural de grau leve; $3 \%$ perda auditiva mista de grau leve; $7 \%$ perda auditiva sensorioneural de grau moderado; $14 \%$ perda auditiva sensorioneural de grau severo; $2 \%$ perda auditiva sensorioneural de grau profundo. Entre estes indivíduos também foi possível aplicar o teste de Risco Relativo, entretanto, como nesta faixa etária não foram encontrados indivíduos com limiares normais, a técnica foi aplicada entre os dados de limiares normais com rebaixamento das frequências de 6000 e $8000 \mathrm{~Hz}$ e perda auditiva. A partir destes dados obtiveram-se os seguintes resultados: $63,64 \%$ de redução absoluta do risco, ou seja, indivíduos nesta faixa etária têm $63,64 \%$ de chances de terem perda auditiva e não apenas limiares normais com rebaixamento de frequências.

Entre os indivíduos entre 80 e 89 anos (3 pessoas/ 6 orelhas) verificou-se $17 \%$ de perda auditiva sensorioneural de grau leve; $50 \%$ de perda auditiva sensorioneural de grau moderado e $33 \%$ de perda auditiva sensorioneural de grau severo. Como a amostra foi considerada muito pequena a probabilidade de erro torna-se grande e o teste Quiquadrado aplicado não obteve resultados significativos. O mesmo ocorreu com a faixa etária acima de 90 anos que apesar de apresentar $50 \%$ de perda auditiva sensorioneural e $50 \%$ de perda auditiva sensorioneural de grau moderado foram utilizados amostra de apenas 4 orelhas. Em ambas pode-se dizer que houve diferença, mas não houve diferença estatística.

Com exceção dos resultados obtidos nos dois indivíduos na amostra acima de 90 anos, quando comparado os graus de perda auditiva entre as diferentes faixas etárias, pode-se observar uma progressiva piora nos limiares auditivos.

A amostra total da pesquisa também foi classificada em hipertensos e não hipertensos. 59,01\% (36 pessoas) apresentaram hipertensão arterial. Desta amostra de hipertensos, $88,88 \%$ (32 pessoas) apresentaram perda auditiva. A partir destes dados, aplicou-se o teste de Risco Relativo entre hipertensos com perda auditiva e hipertensos sem perda auditiva, obtendo os seguintes resultados: $77,78 \%$ de aumento absoluto do risco, sendo este um aumento de 8 chances a mais de hipertensos apresentarem perda auditiva (Tabela 4).

A diabetes mellitus também foi categorizada. $32,78 \%$ da amostra apresentaram diabete mellitus (20 pessoas). Desta amostra de diabéticos, 90\% (18 pessoas) apresentaram perda auditiva. Com 
Tabela 4 - Perda Auditiva e Hipertensão Arterial, Diabetes Mellitus, Alcoolismo e Fumo

\begin{tabular}{lcc}
\hline \multicolumn{3}{c}{ Perda Auditiva e Fatores de Risco } \\
\hline Classificação & Indivíduos & Porcentagem \\
\hline Perda Auditiva e Hipertensão Arterial & 61 & $100 \%$ \\
Amostra total & 36 & $59,01 \%$ \\
Hipertensos & 32 & $88,88 \%$ \\
Hipertensos com perda auditiva & & \\
Perda Auditiva e Diabetes Mellitus & 61 & $100 \%$ \\
Amostra total & 20 & $32,78 \%$ \\
Diabéticos & 18 & $90,00 \%$ \\
Diabéticos com perda auditiva & & \\
Perda Auditiva e Consumo Alcoólico & 61 & $100 \%$ \\
Amostra total & 13 & $21,31 \%$ \\
Alcoólicos & 12 & $92,30 \%$ \\
Alcoólicos com perda auditiva & & \\
Perda Auditiva e Fumo & 61 & $100 \%$ \\
Amostra total & 31 & $51,70 \%$ \\
Fumantes, ex-fumantes ou fumantes passivos & $37,09 \%$ \\
\hline Fumantes, ex-fumantes ou fumantes passivos com perda auditiva & 27 & $87 \%$ \\
\hline
\end{tabular}

estes dados, aplicou-se o teste de Risco Relativo entre diabéticos com perda auditiva e diabéticos sem perda auditiva, obtendo os seguintes resultados: $80 \%$ de aumento absoluto do risco, sendo este um aumento de 9 chances a mais de diabéticos apresentarem perda auditiva (Tabela 4).

O fator de risco álcool também foi verificado. $21,31 \%$ (13 pessoas) do total da amostra declararam consumir álcool com ou sem embriaguez diariamente, 2 a 4 vezes por semana ou semanalmente. Destes consumidores de álcool, 92,30\% (12 pessoas) apresentaram perda auditiva. Com estas informações, foi aplicado o teste de Risco Relativo, onde se verificou um aumento absoluto do risco de $84,62 \%$. Os indivíduos consumidores de álcool apresentaram 12 chances a mais de ter perda auditiva comparado com os que não consomem álcool (Tabela 4).

Outro fator de risco também comparado com perda auditiva foi o fumo. Destes, $51,7 \%$ (31 pessoas) foram classificados como fumantes, ex-fumantes ou fumantes passivos com contato diário com fumantes. Destes indivíduos com contato direto com o fumo, $87,09 \%$ (27 pessoas) apresentaram perda auditiva. Através do teste de Risco Relativo, observou-se que fumantes, ex-fumantes e fumantes passivos com contato diário com o fumo apresentaram maior associação com presença de perda auditiva com 9 chances a mais de ter perda auditiva. $\mathrm{O}$ aumento do risco absoluto foi de $77,42 \%$ ( Tabela 4).

\section{DISCUSSÃO}

Durante o processo de envelhecimento as doenças crônicas aumentam e entre elas está a perda auditiva. Alguns estudos justificam que a alteração no ouvido ocorrida com o passar da idade tem relação com uma insuficiência microcirculatória decorrente de uma oclusão vascular por embolia, hemorragia ou vasoespasmo e que estes por sua vez, seriam decorrentes de uma síndrome de hiperviscosidade ou microangiopatia, sendo que as alterações sistêmicas ou determinados estilos de vida podem vir a acelerar o processo de degeneração do aparelho auditivo. Entre elas estão à inalação de substâncias tóxicas, certas alterações circulatórias e metabólicas além de infecções, traumas de várias naturezas e hereditariedade ${ }^{15}$.

O fator idade foi um importante preditor tanto nas análises brutas como nas ajustadas. Neste trabalho observou-se que após 70 anos não foram encontrados limiares auditivos normais e que após 80 anos também não foram encontrados limiares normais com frequências rebaixadas, indicando que a prevalência de perda auditiva aumenta progressiva e proporcionalmente ao aumento da idade. Diversos estudos têm encontrado resultados semelhantes ${ }^{1,5,31-33}$.

A partir da amostra estudada também se pode concluir que há prevalência da perda auditiva do tipo sensorioneural em relação às demais perdas 
em indivíduos com mais de 60 anos. Esta afirmação pode ser validada através de estudos anteriores ${ }^{2,10,34}$ que também constataram a prevalência deste tipo de perda. Neste estudo, contudo, não foi encontrado nenhum achado audiométrico de perda do tipo condutiva. Apenas dois pacientes apresentaram perda do tipo mista, sendo ambos unilaterais.

No que se refere à Hipertensão arterial este estudo indica a associação entre este fator e perda auditiva. Alguns autores ressaltam que todas as células do corpo dependem de um fornecimento adequado de oxigênio e nutrientes para manterem suas funções, dependendo da integridade funcional e estrutural do coração e dos vasos sanguíneos. A hipertensão pode facilitar alterações do coração e dos vasos sanguíneos. A pressão elevada no sistema vascular pode resultar em hemorragias na orelha interna, podendo levar às perdas auditivas súbitas ou progressivas ${ }^{15,28}$. A Hipertensão é, portanto, um aspecto pertinente aos programas de saúde, pois predispõe ao surgimento de diversas morbidades, inclusive da perda auditiva.

Quanto a diabetes mellitus e a amostra analisada, é possível constatar a associação entre perda auditiva e esta alteração metabólica. Todavia, devido à pequena quantidade de pessoas que apresentaram esta alteração, não é possível assegurar que exista uma efetiva associação entre elas. A literatura acerca deste tema ainda é controversa ${ }^{18,19}$, por isso, faz-se necessário a execução de outras pesquisas a respeito deste assunto para futuros resultados mais significantes.

$\mathrm{Na}$ literatura também se cita que fumantes e ex-fumantes estão mais predispostos a desenvolverem perda auditiva ${ }^{22}$ e também que o consumo excessivo de álcool pode predispor a perda auditiva ${ }^{20,21}$. Neste presente estudo, estes fatores foram efetivamente associados à perda auditiva. Contudo, não se pode considerá-los isoladamente como fatores causais devido à faixa etária estudada e sua associação com a presbiacusia.

Portanto, os fatores de risco, hipertensão arterial, diabetes mellitus, fumo e álcool, abordados neste estudo, obtiveram significante associação estatística com perda auditiva $(p<0,0001)$ quando comparados em teste de Risco Relativo, mas, devido ao pequeno número de indivíduos que apresentaram estes fatores, seria imprudente afirmar a relação direta dos mesmos enquanto causalidade das alterações auditivas encontradas. Outro fator que deve ser ressaltado é a influência do processo presbiacúsico verificado nesta pesquisa, que impede a obtenção clara do fator etiológico principal do desenvolvimento da perda auditiva.

Com isto, conclui-se neste estudo que a perda auditiva sensorioneural é o principal tipo de perda auditiva encontrada em indivíduos com mais de 60 anos de idade, sendo esta perda progressivamente aumentada proporcional ao envelhecimento. Os fatores de risco hipertensão arterial, diabetes mellitus, consumo alcoólico e fumo são fatores que predispõem as perdas auditivas, principalmente quando associados com a presbiacusia.

Espera-se que os dados obtidos com este estudo possam contribuir com estratégias preventivas de saúde, divulgando-se os riscos do álcool, do fumo, da hipertensão arterial e da diabetes mellitus para o desenvolvimento de perda auditiva, bem como o incentivo ao acompanhamento da saúde periódico de idosos no que se refere à audição. Medidas profiláticas que compensem o envelhecimento também podem auxiliar no controle da perda auditiva e na diminuição do impacto psicossocial das alterações auditivas nestes indivíduos.

\section{CONCLUSÃO}

A partir desta pesquisa verificou-se a prevalência de $63,93 \%$ de perda auditiva sensorioneural em indivíduos com mais de 60 anos de idade e uma progressiva piora nos limiares auditivos com o aumento da idade. Também se verificou associação estatisticamente significante entre perda auditiva e os fatores de risco hipertensão arterial, diabetes mellitus, consumo alcoólico e fumo. Entretanto, estes fatores requerem pesquisas mais aprofundadas para a confirmação de sua influência nas alterações auditivas. 


\section{ABSTRACT}

Purpose: to estimate the prevalence of hearing loss $(\mathrm{HL})$, identifying the factors associated with elderly patients in the city of Londrina - PR. Methods: cross-sectional study in a population sample with 61 individuals aged over 60 years in Londrina-Pr, 2009, through anamnesis and audiological evaluation. We used the chi-square of relative risk with $95 \%$ confidence and $p$-value $<0.05$ for univariate analysis between $\mathrm{HL}$ and associated factors. Results: the whole sample $87.70 \%$ had some type of hearing loss or lowering of frequencies. Out of these individuals with $\mathrm{HL}$, the type of $\mathrm{HL}$ with greater prevalence in this age group was sensorineural $\mathrm{HL}$, with $63.93 \%$ of occurrence. Out of the sample of hypertensive patients, $88.88 \%$ had $\mathrm{HL}$, which is an increase of 8 chances to make the hypertensive population to have HL. From the sample of diabetics, $90 \%$ had HL, an increase of 9 chances to cause the diabetic population to show HL. Consumers of alcohol, $92.30 \%$ had HL. Thus, consumers of alcohol had 12 more chances to get HL. However, from the sample of smokers, former smokers or passive smokers with daily contact with smokers, from these individuals, $87.09 \%$ had $\mathrm{HL}$, thus, an increase of 9 chances to have HL. Conclusion: the study showed that people over 60 years shows mainly sensory neural $\mathrm{HL}$, and it is a progressive loss and its proportion increased with aging. Risk factors, such as hypertension, diabetes, alcohol consumption and smoking are factors that can predispose to $\mathrm{HL}$, especially when associated with presbycusis.

KEYWORDS: Hearing Loss; Risk Factors; Cross-Sectional Studies

\section{REFERÊNCIAS}

1. Baraldi GS, Almeida LC, Borges ACC. Evolução da perda auditiva no decorrer do envelhecimento. Rev Bras Otorrinolaringol. 2007; 73(1): 64-70.

2. Greco MC, Russo ICP. Achados audiológicos em indivíduos idosos atendidos em uma clínica particular de São Paulo-SP. Acta ORL. 2006; 24(4): 245-54.

3. Scazufca M, Cerqueira ATAR, Menezes $P R$, Prince $M$, Vallada HP, Miyazaki MCOS. Investigações sobre demências nos países em desenvolvimento. Rev Saúde Publica. 2002; 36(6): 773-8.

4. Carmo LC, Silveira JAM, Marone SAM, D'Ottaviano FG, Zagati LL, Lins EMDS. Estudo audiológico de uma população idosa brasileira. Rev Bras Otorrinolaringol. 2008; 74(3): 342-9.

5. Nobrega JD, Câmera MFS, Borges ACC. Audição do idoso: análise da percepção do prejuízo auditivo, perfil audiológico e suas correlações. RBPS. 2008; 21(1): 39-46.

6. Versas RP, Mattos LC. Audiologia do envelhecimento: revisão da literatura e perspectivas atuais. Rev Bras Otorrinolaringol. 2007; 73(1): 128-34.

7. IBGE. Instituto Brasileiro de Geografia e Estatística. [homepage na internet] Brasília: 2009. [acesso em 18 ago 2009]. Disponível em: URL: http://www.ibge.com.br.

8. Tavares DMS, Guidetti GECB, Saúde MIBM. Características sócio-demográficas, condições de saúde e utilização de serviços de saúde por idosos. Rev.eletr. Enf. [Internet]. 2008; 10(2): 299-309.

9. Dados-Londrina. Saúde em Londrina vacinou 85\% dos idosos. [homepage na internet] Local: Londrina ano 2009. [acesso em 18 ago 2009] Disponível em: URL: http://www.londrix.com.br.

10. Silva BSR, Sousa GB, Russo ICP, Silva JAPR. Caracterização das queixas, tipo de perda auditiva e tratamento de indivíduos idosos atendidos em uma clínica particular de Belém-PA. Arq. Int. Otorrinolaringol. 2007; 11(4): 387-95.

11. Kano CE, Mezzena LH, Guida HL. Estudo comparativo da classificação do grau de perda auditiva em idosos institucionalizados. Rev. CEFAC. 2009; 11(3): 473-77.

12. Russo IP. Intervenção fonoaudiológica na terceira idade. Revinter: Rio de Janeiro; 2004.

13. Valete R, Cláudia M, Rozenfeld S. Triagem auditiva em idoso: comparação entre auto-relato e audiometria. Rev Bras Otorrinolaringol. 2005; 71(2):193-200.

14. ASHA. American Speech, Language and Hearing Association. Hearing Loss. [homepage na internet] EUA: 2009 [acesso em 20 set 2009] Disponível em: URL: http://www.asha.org.

15. Marchiori LLM, Rego FEA, Matsuo T. Hipertensão como fator associado à perda auditiva. Rev Bras Otorrinolaringol. 2006; 72(4): 533-40.

16. Baraldi GS, Almeida LC, Borges ACC. Perda auditiva e hipertensão: achados em grupo de idosos. Rev Bras Otorrinolaringol. 2004; 70(5): 640-4. 
17. Mondelli MFCL, Andréa C. Relação entre a hipertensão arterial e a deficiência auditiva. Arq. Int. Otorrinolaringol. 2009; 13(1): 63-8.

18. Maia CAS, Campos CAH. Diabetes mellitus como causa de perda auditiva. Rev Bras Otorrinolaringol. 2005; 71(2): 208-14.

19. Marchiori LLM, Gibrin PC. Diabetes mellitus: prevalência de alterações auditivas. Arq. Bras. Endocrinol. Metab. 2003; 74(1):82-6.

20. Rossi AG, Bellé M, Sartori SA. Avaliação audiológica básica em alcoólicos. Rev Ci. Méd. Biol. 2006; 5(1):21-8.

21. Ribeiro SBA, Jacob-Corteletti LCB, Alvarenga KF, Marques JM, Campelo RM, Tschoeke SN. Avaliação auditiva em alcoolistas abstêmicos. Rev Bras Otorrinolaringol. 2007; 73(4): 452-62.

22. Angrisani RMG, Matas CG, Furtado JRB. Análise dos potenciais evocados auditivos em fumantes. Acta ORL. 2008; 26(3):146-50.

23. XZ Liu, D Yan. Aging and hearing loss. J Pathol. 2007; 211:188-97.

24. Baraldi GS, Almeida LC, Borges ACC. Hearing loss in aging. Rev Bras Otorrinolaringol. 2007; 73(01): 64-70.

25. Momensohn-Santos TM, Russo IP, organizadores. Prática da audiologia clínica. 6. ed. São Paulo: Cortez; 2007.

26. Stenklev NC, Laukli E. Presbyacusis-hearing thresholds and the ISO 7029. Int J Audiol. 2004; 43:295-306.
27. Spidurso WW. Dimensões físicas do envelhecimento. Barueri: Manole; 2005.

28. Katz J. Tratado de audiologia clínica. São Paulo: Manole; 1989.

29. Gorga MP, Neely ST, Dorn PA. Distortion product otoacoustic emission test performance a priori criteria and for multifrequency audiometric standards. Ear Hear. 1999; 20(40):345-62.

30. Marchiori LLM, Rego Filho EA. Queixa de vertigem e hipertensão arterial. Rev. CEFAC. 2007; 9(1): 116-21.

31. Teixeira AR, Freitas CLR, Millão LF, Gonçalves AK, Becker JB, Vieira AF. Relação entre deficiência auditiva, idade, gênero e qualidade de vida de idosos. Arq. Int. Otorrinolaringol. 2008; 12(1): 62-70. 32. Sousa MGC, Russo ICP. Audição e percepção da perda auditiva em idosos. Rev Soc Bras Fonoaudiol. 2009; 14(2): 241-6.

33. Narciso ÂR, Garbúggio EZ, Sarri EM, Muraro MD, Lopes TA, Oliveira VA. Caracterização da presbiacusia em uma população de idosos. Iniciação científica - Cesumar. 2002; 04(01): 37-49. 34. Patterson C. Prevention of hearing impairment and disability in the elderly. In: Canadian Task Force on the Periodic Health Examination. Canadian Guide to Clinical Preventive Health Care. Ottawa: Health Canada; 1994.p. 954-63.

RECEBIDO EM: 24/11/2009

ACEITO EM: 17/02/2010

Endereço para correspondência:

Luciana Lozza de Moraes Marchiori

Campus Universitário de Londrina - Clinica de

Fonoaudiologia

Av. Paris, 675

Londrina - PR

CEP 86041-140 Cx. P. 401-

E-mail: luciana.marchiori@unopar.br 